\title{
Real-time phase-only color holographic video display system using LED illumination
}

\author{
Fahri Yaraş, ${ }^{*}$ Hoonjong Kang, and Levent Onural \\ Department of Electrical and Electronics Engineering, Bilkent University, TR-06800 Ankara, Turkey \\ ${ }^{*}$ Corresponding author: fahri@ee.bilkent.edu.tr
}

Received 23 June 2009; revised 15 September 2009; accepted 15 September 2009; posted 16 September 2009 (Doc. ID 113153); published 29 September 2009

\begin{abstract}
A real-time full-color phase-only holographic display system generates holograms of 3D objects. The system includes a 3D object formed by voxels, an internet-based transmission capability that transmits the object information to the server, a real-time hologram generation unit, and a holographic display unit with incoherent illumination. The server calculates three phase holograms for RGB components using multiple GPUs. The resultant phase holograms are saved into an RGB bitmap image and loaded to the phase-only spatial light modulators (SLMs). SLMs are illuminated uniformly by LEDs, and reconstructed waves are aligned and overlapped by using high precision optics and stages. Experimental results are satisfactory. (C) 2009 Optical Society of America

OCIS codes: $\quad 090.5694,090.2870,090.4220,090.1705$.
\end{abstract}

\section{Introduction}

Real-time holographic display systems are under investigation for potential 3D TV applications. Realtime holographic fringe pattern generation will have a significant impact on future 3D display technologies. Unfortunately there are some severe bottlenecks in fast computation of digital holograms. Methods for fast computation of holograms are mentioned in [1-3]. Some real-time holographic display systems have already been demonstrated. SeeReal, QinetiQ, and the Massachusetts Institute of Technololgy's Holo-video are some of these [4-7].

In our display system we used the Accurate Compensated Phase-Added Stereogram (ACPAS) [8]. This algorithm is proposed by Kang and can be use $\bar{d}$ as color holographic fringe generation method. ACPAS is an enhanced version of the Compensated PhaseAdded Stereogram (CPAS) [9]. The computation time of ACPAS is slightly longer than the CPAS, but it gives satisfactory results that are similar to those of Fresnel holograms. We used phase-only spatial light modulators (SLMs) to display holograms, and we benefit

$0003-6935 / 09 / 340 \mathrm{H} 48-06 \$ 15.00 / 0$

(C) 2009 Optical Society of America from the desirable properties of phase holograms. These properties, such as low-power diffraction orders, high diffraction efficiency and a low-power undiffracted beam, are listed in $[10,11]$. To illuminate those SLMs we used light-emitting diodes (LEDs). With the help of LEDs, we eliminate the adverse effects of lasers such as speckle noise and eye hazard. When coherent light passes through or reflects back from randomly diffused media, randomized phase regions are generated and an interference pattern is observed due to those random phase regions; the resultant noisy pattern is called "speckle noise" [12]. There are many methods to eliminate this undesirable effect [13-17], however, none of them seems to be suitable for real-time holography. LEDs have both time and space coherence to some extent. Narrow spectrum of LEDs brings a time coherence and using a pinhole in front of them increases the spatial coherence. However, since LEDs do not generate fully coherent light, there is no speckle noise. LEDs are not harmful as lasers if they are not too bright [18]. Therefore reconstructed images can be observed by the naked eye. Other advantages of LEDs are ease of operation and their low cost. However due to low coherence characteristics reconstructions might be 
somewhat blurred. Holographic reconstructions using phase-only SLMs and LEDs were reported in [19-23].

To achieve real-time generation of full color fringe patterns, we used a multi-GPU system. GPUs are powerful processing units that can be used as parallel processing devices [24-26]. Since the ACPAS algorithm is suitable for parallel processing, the multiGPU system speeds up the overall performance, significantly. Our system consists of three major parts: client, server, and optics. In the client, a 3D rigid object is stored. It can be controlled freely by the user, and therefore it brings interactivity to the system. Users can hold the object or rotate it as they like. The model consists of discrete points in space. Each point is represented by its 3D coordinates and color values. For a specified volume, coordinate and color information associated with all points that make up the 3D scene is sent to the server by the TCP/IP protocol. The server calculates three holograms by using the multi-GPU architecture for red, green, and blue components of the 3D scene upon receiving the data. Resultant holograms are output as an RGB bitmap image and directed to phase-only SLMs by the computer graphics unit. After loading red, green, and blue holograms to the SLMs, they are illuminated by the uniform light beams that are propagated from LEDs (Fig. 1). When the light hits the SLMs, each pixel modulates the phase and reflects the light back. With the help of beam splitters and high precision microstages, red, green, and blue waves are overlapped. Reconstructed images are captured by a CCD camera.

\section{Algorithm}

In order to achieve real-time holographic display, a fast digital hologram generation method is needed. One of the fast methods is the coherent holographic stereogram [9]. The ACPAS $[\underline{8}, 21,22,27,28]$ is the latest improvement of the coherent holographic stereo-

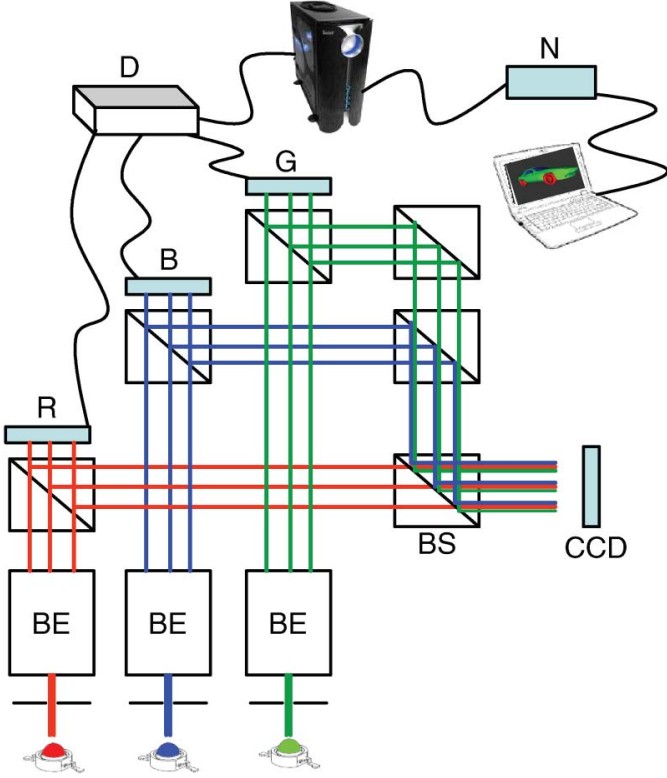

Fig. 1. (Color online) Overall setup: BE, beam expander; $\mathrm{R}$, red SLM; B, blue SLM; G, green SLM; D, driver unit of SLMs; N, network; BS, nonpolarized beam splitters.

gram. The reconstruction from the ACPAS closely resembles the reconstruction from the Fresnel hologram, and computation using the ACPAS is much faster than the computation of the Fresnel hologram. Therefore, the ACPAS is applicable for a practical interactive system.

The geometry of coherent stereogram calculation is shown in Fig. 2. The ACPAS algorithm first starts with a blank $2 \mathrm{D}$ plane. Then it is partitioned into suitable square size segments. Within a segment, contribution of each object point in the point cloud is approximated as a single complex sinusoid weighted by the corresponding amplitude; $\left(a_{i} / r_{i}\right) \exp \left(j k r_{i}\right) \times$

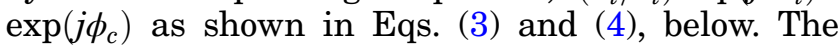

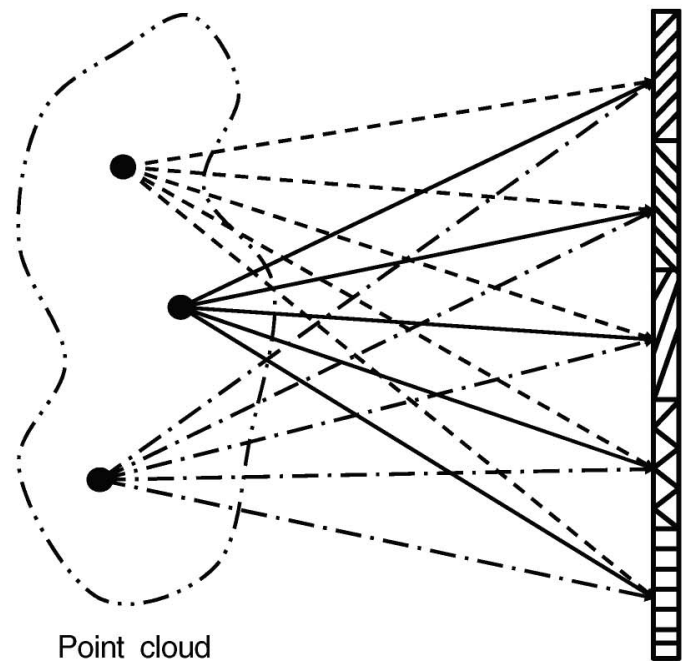

Hologram

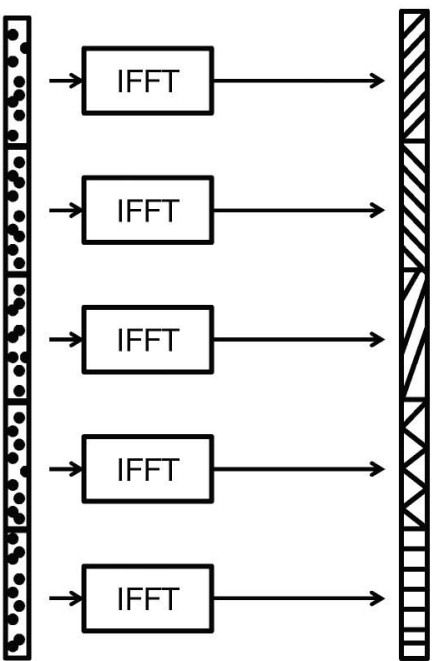

Spectrum

Hologram

Fig. 2. Hologram calculation algorithm. 


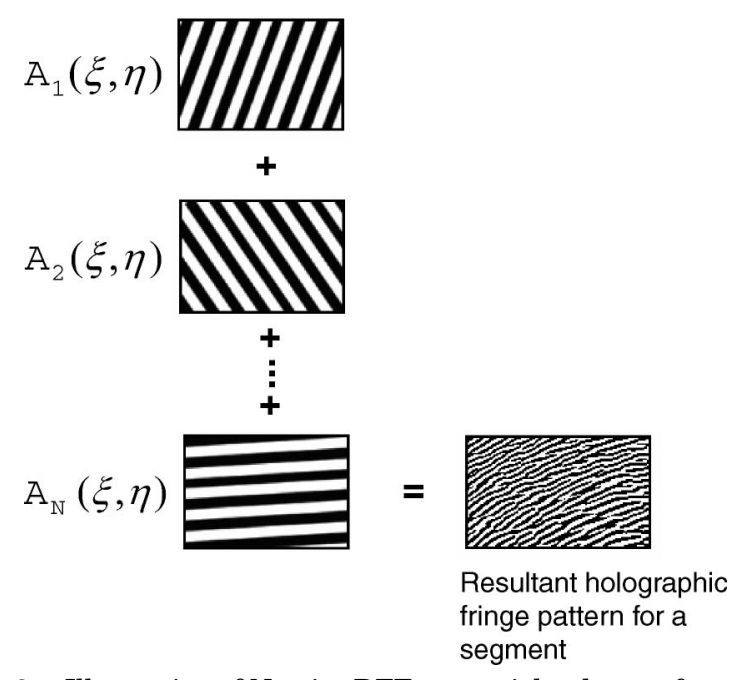

Fig. 3. Illustration of $N$-point DFT as a weighted sum of complex sinusoids.

holographic fringe pattern for a single segment in the $(\xi, \eta)$ plane using a point cloud in $(x, y, z)$ volume is expressed as []

$$
\begin{aligned}
\mathbf{I}_{\mathrm{ACPAS}}(\xi, \eta)= & \sum_{i=1}^{N} \frac{a_{i}}{r_{i}} \exp \left\{j 2 \pi \left[\left(\xi-\xi_{c}\right) f_{i \xi c \mathrm{int}}\right.\right. \\
& \left.\left.+\left(\eta-\eta_{c}\right) f_{i \eta \mathrm{int}}\right]+j k r_{i}+j \phi_{c}\right\},
\end{aligned}
$$

where $N$ is the number of object points, $a_{i}$ is the intensity of an object point, and the wavenumber $k$ is $2 \pi / \lambda$, where $\lambda$ is the free-space wavelength of the coherent light. The distance $r_{i}$ between the $i$ 'th object point and the point $\left(\xi_{c}, \eta_{c}\right)$ on the hologram is $\left[\left(\xi_{c}-x_{i}\right)^{2}+\left(\eta_{c}-y_{i}\right)^{2}+z_{i}^{2}\right]^{1 / 2}$. The $\left(\xi_{c}, \eta_{c}\right)$ is the center coordinate of each segment on the hologram. The related spatial phase error compensation $\phi_{c}$ is determined as []

$$
\phi_{c}=2 \pi\left\{\left(f_{i \xi c}-f_{i \xi \text { int }}\right)\left(\xi_{c}-x_{i}\right)+\left(f_{i \eta c}-f_{i \eta c \mathrm{int}}\right)\left(\eta_{c}-y_{i}\right)\right\},
$$

where $f_{i \xi c}$ and $f_{i \eta c}$ are the continuous spatial frequencies and $f_{i \xi c i n t}$ and $f_{i \eta \text { cint }}$ are discrete spatial frequencies in cycles per unit length on the $\xi$ and $\eta$ axes, respectively. For a given segment we can simplify Eq. (1) as

$$
\mathbf{I}_{\mathrm{ACPAS}}(\xi, \eta)=\sum_{i=1}^{N} A_{i}(\xi, \eta) \exp \left(j 2 \pi \Phi_{i}(\xi, \eta)\right),
$$

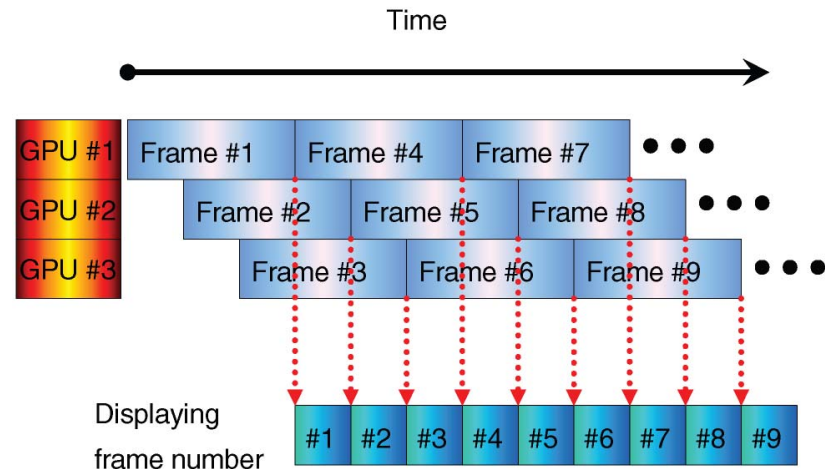

Fig. 4. (Color online) Pipelined computation using GPUs.

where

$$
\begin{gathered}
A_{i}(\xi, \eta)=\frac{a_{i}}{r_{i}} \exp \left(j k r_{i}\right) \exp \left(j \phi_{c}\right), \\
\Phi_{i}(\xi, \eta)=\left(\xi-\xi_{c}\right) f_{i \xi \mathrm{cint}}+\left(\eta-\eta_{c}\right) f_{i \eta \mathrm{cint}} .
\end{gathered}
$$

With the help of this simplification, we see that Eq. (3) is an $N$-point inverse discrete Fourier transform (within a trivial constant gain) and an inverse fast Fourier transform is used for its computation. To calculate the resultant fringe pattern, each segment can be processed independently by this method. This makes the ACPAS algorithm suitable for parallel processing. Figures 2 and 3 show the hologram computation algorithm. The computed fringe pattern is then written onto magnitude-only, phase-only, or combined (magnitude and phase) SLMs since the fringe pattern is a complex valued function. In our case, we just extracted and used the phase information from this complex field by assigning a constant value to the magnitude and used a phase-only SLM.

\section{Client}

The 3D model of the object is stored in the client computer (Fig. 1). Our rigid model consists of discrete points that are floating in space. The model can be controlled by the peripherals of the computer (by mouse or keyboard) so that the object can be moved or rotated in every direction or held still. OpenGL is used to display the model in the client computer. The $3 \mathrm{D}$ coordinates of the object points are updated as the object moves. Then for each frame of the 3D scene, all information (3D coordinates and red, green, blue color information associated with each object point) is sent to the server through the internet. However since there is no feedback, if the server could not

Table 1. Overall System Specifications

$\begin{array}{lll}\text { Computing system } & \text { CPU } & \text { Two Intel(R) Xeon(R) CPU 2 GHz } \\ & \text { Main memory } & \text { 8 Gbits } \\ \text { Programming environment } & \text { GPU } & \text { Three NVIDIA GeForce GTX 280 } \\ & \text { Operating system } & \text { Linux 64 bit (Ubuntu 8.10) } \\ & \text { Programming language } & \text { Standard C and CUDA } \\ & \text { Libraries } & \text { CUFFTW }\end{array}$




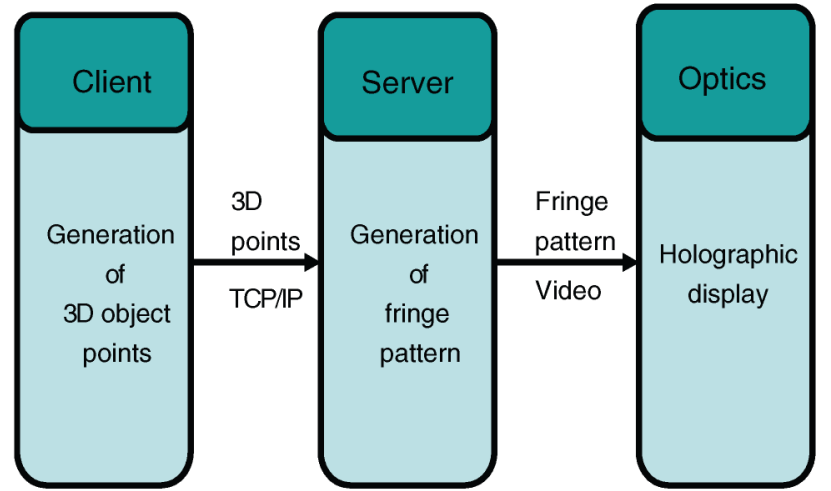

Fig. 5. (Color online) End-to-end system.

finish the hologram generation of the previous frame, data packages that are received from client are dropped. Since there is no computational load on this stage, an average PC or a laptop can be used as a client.

\section{Server}

Computer generated holograms are calculated by the server computer (Fig. 1). The object point information that is sent by the client is received by the server. Upon receiving the information, the holographic fringe pattern over each segment is calculated as described in Section 2. As a consequence of the segmented structure, the inverse fast Fourier transform of each segment is calculated in parallel by using the GPU architecture. This speeds up the overall performance. Moreover, in order to increase the frame rate, we use multiple GPUs. Three GPU boards are used to achieve a pipelined real-time computation. As shown in Fig. 4, while first GPU calculating the $n$th frame of the scene, second and third GPUs are processing the $(n+1)$ st and $(n+2)$ nd frames, respectively. Theoretically we expect to triple the frame rate. This hologram generation process is executed for each color separately. Then the phase of the each complex field is extracted. After phase holograms for the red, green, and blue components of the 3D scene are obtained, they are saved into a single RGB bitmap image by mapping the phase values ( 0 to $2 \pi$ $\mathrm{rad}$ ) to grayscale (0 to 255). The computer graphics unit of the server then outputs the resultant RGB image to the driver unit of the SLMs. Table $\underline{1}$ shows the overall system specifications of the server.

Table 2. Characteristics of LEDs

\begin{tabular}{llcc}
\hline Part Number & Color & Emission Angle $^{a}$ & Wavelength $(\mathrm{nm})^{\circ}$ \\
\hline EDER-1LA3 & Red & $120^{\circ}$ & $620-630$ \\
EDET-1LA2 & Green & $150^{\circ}$ & $515-535$ \\
EDEB-1LA5 & Blue & $150^{\circ}$ & $455-475$ \\
\hline
\end{tabular}

${ }^{a}$ Emission angle is the full angle where the intensity of light falls to half of the on-axis intensity (C) 2009 Edison Opto Corporation. Reprinted with permission).
Table 3. Performance Analysis of the System for 2 Mpixel Hologram Output [22]

\begin{tabular}{cccc}
\hline $\begin{array}{c}\text { No. of } \\
\text { Object Points }\end{array}$ & $\begin{array}{c}\text { One GPU } \\
\text { (fps) }\end{array}$ & $\begin{array}{c}\text { Two GPUs } \\
\text { (fps) }\end{array}$ & $\begin{array}{c}\text { Three GPUs } \\
\text { (fps) }\end{array}$ \\
\hline 1 & 15.8 & 31.6 & 47.5 \\
10 & 15.7 & 31.4 & 47.2 \\
100 & 15.3 & 30.7 & 46.1 \\
1000 & 13.5 & 27.0 & 40.5 \\
10000 & 6.8 & 13.6 & 20.5 \\
\hline
\end{tabular}

\section{Optics}

The last part of the system is the optics (Fig. 5). In this stage there are LEDs, beam expanders, beam splitters (beam combiners), phase-only SLMs, and a CCD camera. The light from each LED first passes through a spatial filter. Each light beam is first focused by $40 \times$ microscope objectives. A pinhole whose diameter is $200 \mu \mathrm{m}$ is located at the focal point of the objective to filter out the high frequency components. This also helps us to increase the spatial coherence of the light. According to our experiments, pinhole diameters between 50 and $500 \mu \mathrm{m}$ give satisfactory results. The light beam that passes through a pinhole is then expanded by the beam expanders to achieve a uniform plane wave illumination. Each color hits the corresponding phase-only SLM, which is already loaded with the phase hologram. We have used Holoeye's HEO1080P phase-only SLMs. They have $1920 \times 1080$ pixels and $8 \mu \mathrm{m} \times 8 \mu \mathrm{m}$ square-shaped pixels. After reflection of the light from the SLMs, each color reconstruction is aligned and overlapped with the help of high precision mechanical stages to yield the final result. We used a CCD camera to record the resultant $3 \mathrm{D}$ image. Table $\underline{2}$ shows the characteristics of LEDs.

\section{Results}

The performance analysis of the system for 2 Mpixel holographic fringe generation is shown in Table 3 . Objects that contain around 10,000 object points

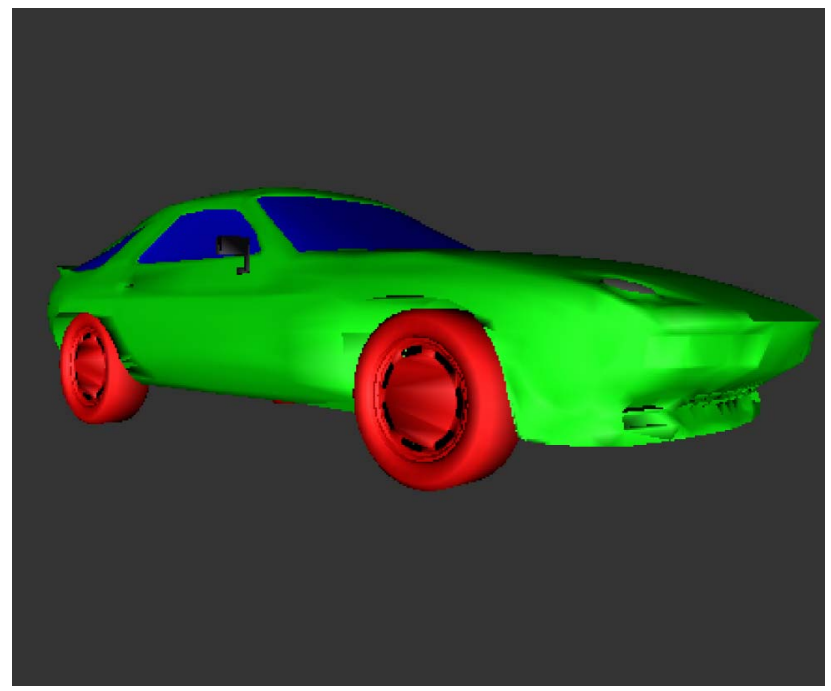

Fig. 6. (Color online) Rigid color 3D object. 


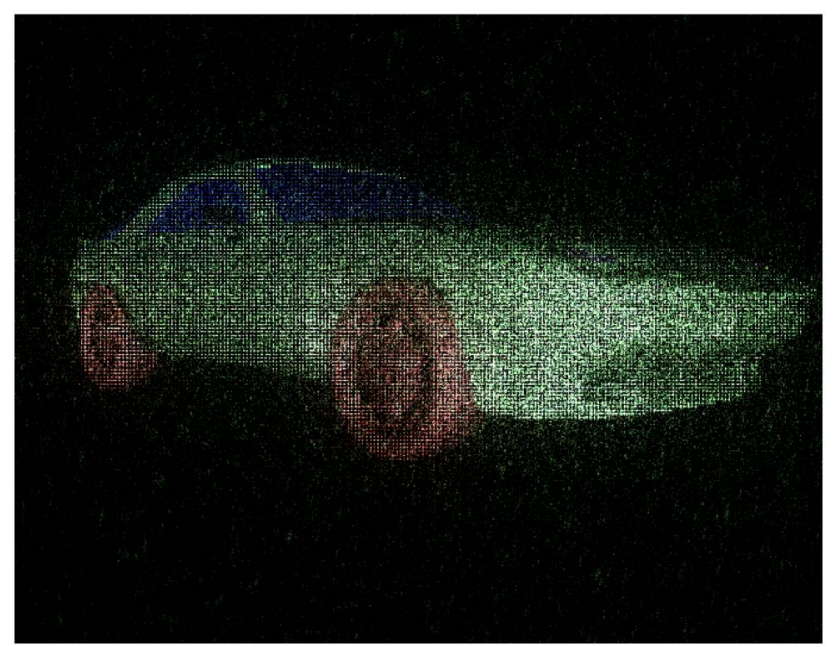

Fig. 7. (Color online) Computer reconstruction using the ACPAS algorithm.

can be displayed in real time by using three GPUs. Moreover we can interpret that there is a linear relationship between the number of GPUs and frame rates. In Fig. $\underline{6}$ and 7 a 3D model and computer reconstruction from $\bar{a}$ hologram computed using ACPAS are shown, respectively. Computer reconstruction is obtained by Fresnel propagation from the computer generated hologram. Monochromatic reconstructions using a green laser and LED are

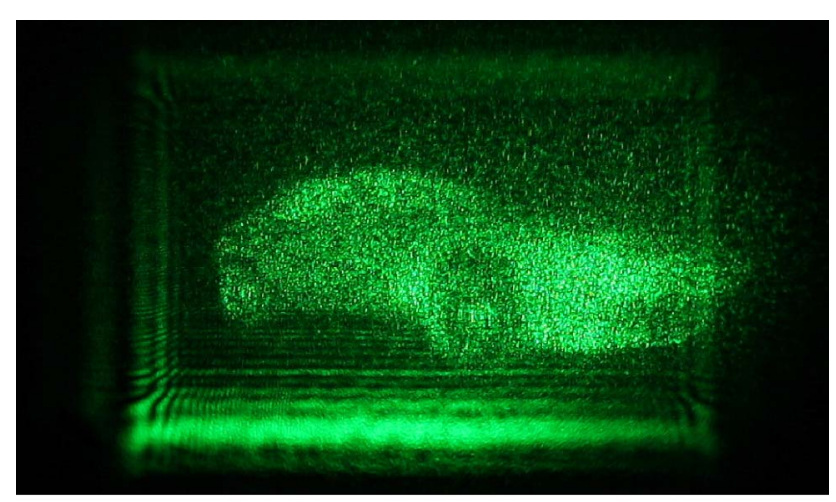

(a)

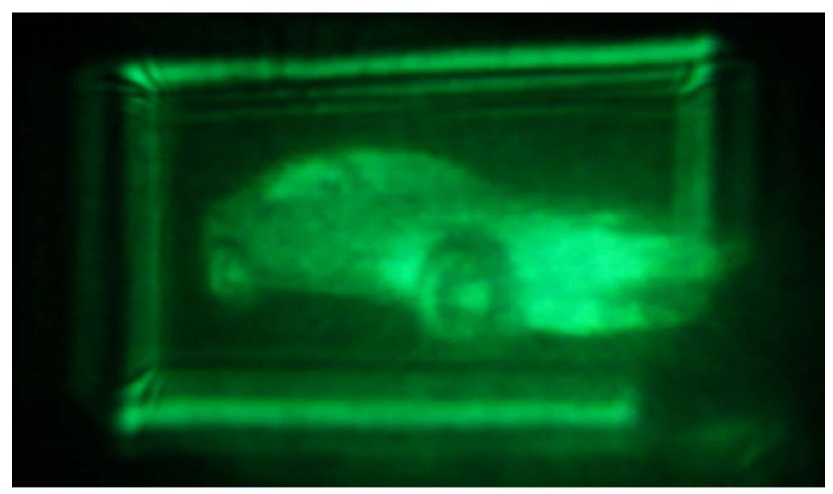

(b)

Fig. 8. (Color online) Single color reconstruction (a) by green laser (b) by green LED.

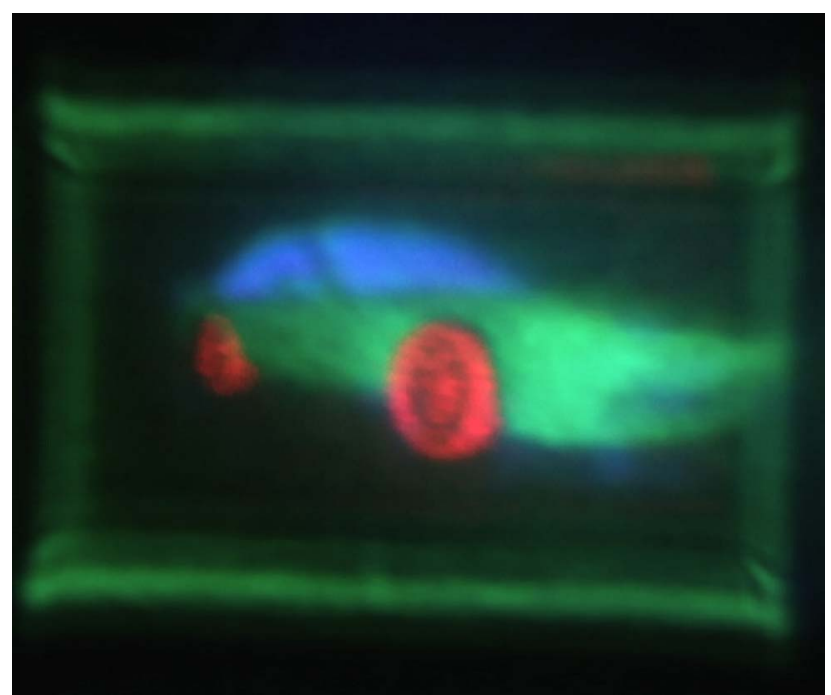

Fig. 9. (Color online) Optical reconstruction of a single frame of the 3D object.

illustrated in Fig. 8 to compare the effect of using low coherence light sources. In Fig. 8(a) speckle noise is observed due to the coherent light source and it degrades the quality. On the other hand reconstruction with LED is satisfactory and speckle noise does not exist (Fig. 8(b)). We have used Edixeon $1 \mathrm{~W}$ point type LEDs, which are manufactured by Edison Opto Corporation. The optical reconstruction of a single frame of the $3 \mathrm{D}$ object, which is recorded as a $2 \mathrm{D}$ photograph using a CCD camera, is shown in Fig. $\underline{9}$. This optical reconstruction is generated using phase holograms that are calculated by using two GPUs.

\section{Conclusions}

It is observed that the proposed system can be used as a color holographic display system. We have shown that ACPAS is an advantageous algorithm for holographic display systems, and it can be computed using multi-GPU environments. Our system also demonstrates that point-based $3 \mathrm{D}$ representation can be transmitted over a network for subsequent real-time holographic fringe pattern generation. Thus it is shown that it is feasible to drive a holographic display by remotely stored $3 \mathrm{D}$ information. We triple the frame rate of the reconstructed $3 \mathrm{D}$ video by using three GPUs. Although LEDs a have wider spectrum, reconstructions are comparable to the coherent case. Since phase holograms are used and since the pixel geometries and properties of the phase-only SLMs are quite good, multiple diffraction orders are almost invisible; this is a feature that improves the reconstruction quality significantly. The reconstructed scene can be seen directly by the naked eye.

This work is supported by the European Commission within FP7 under grant 216105 with the acronym Real 3D.

\section{References}

1. K. Taima, H. Ueda, H. Okamoto, T. Kubota, Y. Nakamura, H. Nishida, H. Takahashi, and E. Shimizu, "New approach to 
the interactive holographic display system," Proc. SPIE 2176 , 23-29 (1994).

2. J. Watlington, M. Lucente, C. Sparrell, V. Bove, and I. Tamitani, "A hardware architecture for rapid generation of electro-holographic fringe patterns," Proc. SPIE 2406, 172183 (1995).

3. H. Yoshikawa and T. Yamaguchi, "Fast hologram calculation for holographic video display," Proc. SPIE 6027, 561-566 (2006).

4. S. Reichelt, R. Haussler, N. Leister, G. Futterer, and A. Schwerdtner, "Large holographic 3D displays for tomorrows TV and monitors-solutions, challenges, and prospects," in IEEE Lasers and Electro-Optics Society, 2008. LEOS 2008, 21st Annual Meeting of the IEEE (IEEE, 2008), pp. 194-195.

5. M. Stanley, M. A. Smith, A. P. Smith, P. J. Watson, S. D. Coomber, C. D. Cameron, C. W. Slinger, and A. D. Wood, "3D electronic holography display system using a 100 mega-pixel spatial light modulator," Proc. SPIE 5249, 297-308 (2004).

6. P. S. Hilaire, S. Benton, M. Lucente, and H. P. M., "Color images with the MIT holographic video display," Proc. SPIE 1667, 73-84 (1992).

7. D. E. Smalley, Q. Y. J. Smithwick, and J. V. M. Bove, "Holographic video display based on guided-wave acousto-optic devices," Proc. SPIE 6488, 64880L (2007).

8. H. Kang, "Quality improvements of the coherent holographic stereogram for natural 3D display and its applications," Ph.D. dissertation (Nihon University, 2008).

9. H. Kang, T. Fujii, T. Yamaguchi, and H. Yoshikawa, "Compensated phase-added stereogram for real-time holographic display," Opt. Eng. 46, 095802 (2007).

10. L. B. Lesem, P. M. Hirsch, and J. J. A. Jordan, "The kinoform: a new wave front reconstruction device," IBM J. Res. Dev. 13, 150155 (1969).

11. C. Kohler, X. Schwab, and W. Osten, "Optimally tuned spatial light modulators for digital holography,” Appl. Opt. 45, 960967 (2006).

12. L. I. Goldfischer, "Autocorrelation function and power spectral density of laser-produced speckle patterns," J. Opt. Soc. Am. 55, 247-252 (1965).

13. H. J. Gerritsen, W. J. Hannan, and E. G. Ramberg, "Elimination of speckle noise in holograms with redundancy," Appl. Opt. 7, 2301-2311 (1968).

14. J. Mark, E. Myers, and A. M. Wims, "Elimination of speckle noise in laser light scattering photometry," Appl. Opt. 11, 947-949 (1972).

15. M. Matsumura, "Speckle noise reduction by random phase shifters,” Appl. Opt. 14, 660-665 (1975).
16. J. Amako, H. Miura, and T. Sonehara, "Specklenoise reduction on kinoform reconstruction using a phaseonly spatial light modulator," Appl. Opt. 34, 3165-3171 (1995).

17. J. M. Huntley and L. Benckert, "Speckle interferometry: noise reduction by correlation fringe averaging," Appl. Opt. 31, 2412-2414 (1992).

18. Y. Barkana and M. Belkin, "Laser eye injuries," Survey Ophthalmol. 44, 459-478 (2000).

19. F. Yaras, M. Kovachev, R. Ilieva, M. Agour, and L. Onural, "Holographic reconstructions using phase-only spatial light modulators," in Proceedings of $3 D$ TV Conference: The True Vision-Capture, Transmission and Display of $3 D$ Video (2008), paper PD-1.

20. F. Yaras and L. Onural, "Color holographic reconstruction using multiple SLMs and LED illumination," Proc. SPIE 7237, 723700 (2009).

21. F. Yaraş, H. Kang, and L. Onural, "Real-time multiple SLM color holographic display using multiple GPU acceleration," in Digital Holography and Three-Dimensional Imaging (Optical Society of America, 2009), paper DWA4.

22. F. Yaras, H. Kang, and L. Onural, "Real-time color holographic video display system," in Proceedings of $3 D T V$ Conference: The True Vision-Capture, Transmission and Display of $3 D$ Video (IEEE, 2009).

23. M. Kovachev, R. Ilieva, P. Benzie, G. B. Esmer, L. Onural, J. Watson, and T. Reyhan, "Holographic 3DTV displays using spatial light modulators," in Three-Dimensional TelevisionCapture, Transmission, Display, H. M. Ozaktas and L. Onural, eds. (Springer, 2008), pp. 529-555.

24. M. Janda, I. Hanák, and L. Onural, "Hologram synthesis for photorealistic reconstruction,” J. Opt. Soc. Am. A 25, 30833096 (2008).

25. N. Masuda, T. Ito, T. Tanaka, A. Shiraki, and T. Sugie, "Computer generated holography using a graphics processing unit," Opt. Express 14, 603-608 (2006).

26. L. Ahrenberg, P. Benzie, M. Magnor, and J. Watson, "Computer generated holography using parallel commodity graphics hardware," Opt. Express 14, 7636-7641 (2006).

27. H. Kang, F. Yaraş, L. Onural, and H. Yoshikawa, "Real-time fringe pattern generation with high quality," in Digital Holography and Three-Dimensional Imaging (Optical Society of America, 2009), paper DTuB7.

28. H. Kang, F. Yaras, and L. Onural, "Quality comparison and acceleration for digital hologram generation method based on segmentation," in Proceedings of 3DTV Conference: The True Vision-Capture, Transmission and Display of $3 D$ Video, (IEEE, 2009). 